\title{
Effect of lincRNA-p21 targeting HIF-1 $\alpha$ on biological functions of liver cancer cells
}

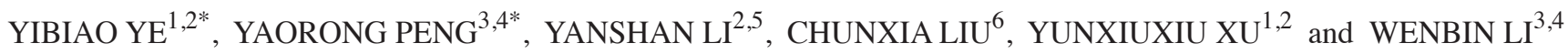 \\ ${ }^{1}$ Department of Hepatobiliary Surgery, ${ }^{2}$ Key Laboratory of Malignant Tumor Gene Regulation \\ and Target Therapy of Guangdong Higher Education Institutes and ${ }^{3}$ Department of Biliopancreatic Surgery, \\ Sun Yat-sen Memorial Hospital, Sun Yat-sen University; ${ }^{4}$ Guangdong Provincial Key Laboratory \\ of Malignant Tumor Epigenetics and Gene Regulation; Departments of ${ }^{5}$ Blood Transfusion and ${ }^{6}$ Ultrasound, \\ Sun Yat-sen Memorial Hospital, Sun Yat-sen University, Guangzhou, Guangdong 510120, P.R. China
}

Received March 28, 2018; Accepted February 5, 2019

DOI: $10.3892 /$ ol.2019.10195

\begin{abstract}
The effects of long intergenic non-coding ribonucleic acid (lincRNA)-p21 targeting hypoxia-inducible factor-1 $\alpha$ (HIF-1 $\alpha)$ on proliferation, apoptosis and migration of liver cancer cells were investigated. MHCC97H liver cancer cells were infected with control lentivirus (control group) and lincRNA-p21 lentivirus (observation group), and control stable cell lines and lincRNA-p21 stable cell lines were screened and obtained by using puromycin. The expression levels of lincRNA-p21 messenger RNA (mRNA) in the control and observation groups were analyzed via reverse transcription-quantitative polymerase chain reaction (RT-qPCR). Bioinformatics was used to search for the lincRNA-p21 target. The expression of target gene was analyzed via western blotting, and the proliferation, apoptosis, migration and in vivo tumor formation of $\mathrm{MHCC} 97 \mathrm{H}$ cells in the control and observation groups were also analyzed. Compared with that in control group, the lincRNA-p21 mRNA level in observation group was increased significantly $(\mathrm{P}<0.05)$. It was found via bioinformatic comparison that HIF-1 $\alpha$ was one of the targets of lincRNA-p21. Results of Western blotting revealed that the expression level of HIF-1 $\alpha$ protein in cells in observation group was significantly downregulated $(\mathrm{P}<0.05)$. Besides, the level of vascular endothelial growth factor (VEGF) protein in cells in control group was obviously higher than that in observation group $(\mathrm{P}<0.05)$. Compared with those in control group, the cell proliferation and migration capacities in observation group were markedly reduced, but the apoptosis level was significantly increased $(\mathrm{P}<0.05)$. According to the in vivo tumor
\end{abstract}

Correspondence to: Dr Wenbin Li, Department of Biliopancreatic Surgery, Sun Yat-sen Memorial Hospital, Sun Yat-sen University, 107 Yanjiang West Road, Guangzhou, Guangdong 510120, P.R. China E-mail: gzsurgeon@163.com

*Contributed equally

Key words: lincRNA-p21, liver cancer, HIF-1 $\alpha$, proliferation, invasion formation assay, the cell proliferation rate in control group was obviously higher than that in observation group $(\mathrm{P}<0.05)$. The number of tumor blood vessels in cells in control group was obviously reduced compared with that in observation group $(\mathrm{P}<0.05)$. lincRNA-p21 can significantly downregulate the level of HIF-1 $\alpha$, thus downregulating the expression of VEGF and affecting the cell proliferation, apoptosis and migration.

\section{Introduction}

Liver cancer is one of the common malignant tumors in clinic, and it is a tumor that affects human health. In particular, primary liver malignant tumors, which originate from the epithelial or mesenchymal tissues of the liver, are high-risk malignant tumors in China. Investigating the pathogenesis of liver cancer from the molecular perspective is currently a hot spot in clinical research. At present, many proteins and RNAs have been found to play an important role in the occurrence, development and metastasis of liver cancer (1-3). Long non-coding ribonucleic acid (lncRNA) is an RNA that is over 200 nucleotides in length, does not encode a protein, and accounts for over $80 \%$ of the total gene (4,5). Current studies have found that IncRNA plays an important role in the proliferation, apoptosis and migration of tumor cells $(6,7)$. Long intergenic non-coding RNA (lincRNA)-p21 of $3.1 \mathrm{~kb}$ in length is one of the lncRNAs, which is transcribed from the antisense strand of p21, and its sequence is highly conserved (8). The low expression level of lincRNA-p21 is closely related to the staging of liver cancer, and can be used to predict the survival time of patients with liver cancer (9). Thus, it can be seen that lincRNA-p21 is of great significance in the occurrence of liver cancer. In this study, the effects of lincRNA-p21 overexpression on the biological behavior of liver cancer cells were analyzed at the cellular and animal levels in vitro.

\section{Materials and methods}

General materials and instruments. The MHCC97H liver cancer cell line was purchased from the American Type Culture Collection (Manassas, VA, USA). lincRNA-p21 lentivirus 
and control lentivirus with a titer of approximately $10^{14}$ were packaged by Shandong Weizheng Biotechnology Co., Ltd. (Shandong, China). Bab/c nude mice, aged 6-8 weeks, were purchased from Beijing Vital River Laboratory Animal Technology Co., Ltd. (Beijing, China). The 2X SYBR-Green quantitative polymerase chain reaction (qPCR) Mix, reverse transcription kit and RNA extraction kit were purchased from Nanjing Vazyme Biotech Co., Ltd. (Nanjing, China). Mouse anti-human hypoxia-inducible factor-1 $\alpha$ (HIF-1 $\alpha)$ monoclonal antibody was purchased from Santa Cruz Biotechnology (cat. no. sc-53546; Santa Cruz, CA, USA). Vascular endothelial growth factor (VEGF) enzyme-linked immunosorbent assay (ELISA) kit was purchased from R\&D Systems, Inc. (Minneapolis, MN, USA). Horseradish peroxidase (HRP)-labeled goat anti-mouse secondary polyclonal antibody was purchased from Hangzhou HuaAn Biotechnology Co., Ltd. (HuaBio) (cat. no. HA1006; Hangzhou, China). Cell Counting Kit-8 (CCK-8) reagent was purchased from Dojindo (Kumamoto, Japan). Real-time PCR amplification instrument was purchased from Bio-Rad Laboratories, Inc. (Hercules, CA, USA). SuPerMax 3000FA Multi-function Microplate Reader was purchased from Shanghai Flash Spectrum Biological Technology Co., Ltd. (Shanghai, China).

The study was approved by the Ethics Committee of Sun Yat-sen Memorial Hospital, Sun Yat-sen University (Guangzhou, China).

Construction of lincRNA-p21 overexpression stable cell lines. After $1 \times 10^{4} \mathrm{MHCC} 97 \mathrm{H}$ cells were inoculated into a 6-well plate and adhered to the wall, the empty vector- and lincRNA-p21 overexpression vector-packaged viruses were used to infect cells. After infection for $48 \mathrm{~h}$, the original medium was replaced with fresh medium. Then unsuccessfully infected cells were killed by using $1 \mu \mathrm{g} / \mathrm{ml}$ puromycin, while infected cells continued to be cultured to restore their normal conditions, followed by amplification. The lincRNA-p21 expression level was detected via reverse transcription-quantitative polymerase chain reaction (RT-qPCR).

Establishment of RT-qPCR system. Control cell lines and lincRNA-p21 overexpression cell lines were collected, and RNA was extracted with TRIzol (Thermo Fisher Scientific, Inc., Waltham, MA, USA) and reverse transcribed into the complementary deoxyribonucleic acid (cDNA) by using the RNA extraction kit and reverse transcription kit. The lincRNA-p21 primers were designed: 21-F, 5' - T TCACTCTGTCCAGGTTG-3' and 21-R, 5' - GA A ACT TATCCCTGACCC-3'; GAPDH-F, 5'-AGAAGGCTGGGGCTCATTTG-3' and GAPDH-R, 5'-AGGGGCCATCCACAGTCTTC-3'. Glyceraldehyde3-phosphate dehydrogenase (GAPDH) was used as an internal reference. PCR system was $21 \mu \mathrm{l}$ in total, including $8 \mu \mathrm{l}$ distilled water, $10 \mu 1$ 2X SYBR-Green qPCR Mix, $1 \mu 1$ 21-F, $1 \mu \mathrm{l} 21-\mathrm{R}$, and $1 \mu \mathrm{l} \mathrm{cDNA}$. Then PCR was set as: $94^{\circ} \mathrm{C}$ for $1 \mathrm{~min}, 94^{\circ} \mathrm{C}$ for $1 \mathrm{~min}, 58^{\circ} \mathrm{C}$ for $30 \mathrm{sec}, 72^{\circ} \mathrm{C}$ for $30 \mathrm{sec}$, a total of 40 cycles. After the end of PCR, agarose gel electrophoresis was performed directly in the PCR system, and the quantitative results were read directly on the instrument using the $2^{-\Delta \Delta \mathrm{Cq}}$ method (10).

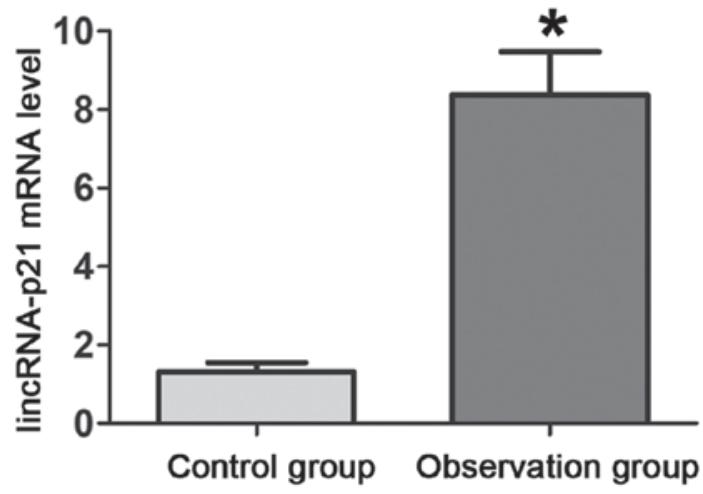

Figure 1. Comparison of lincRNA-p21 mRNA level in cells between the control and observation groups. lincRNA, long intergenic non-coding ribonucleic acid. ${ }^{*} \mathrm{P}<0.05$

Analysis of HIF-1 $\alpha$ protein expression via western blotting. After $1 \times 10^{6}$ control cell lines and $1 \times 10^{6}$ lincRNA-p21 overexpression cell lines in the exponential growth phase were collected, and they were centrifuged at 3,000 x $\mathrm{g}$ for $5 \mathrm{~min}$ at $4^{\circ} \mathrm{C}$. The supernatant was discarded, and cells were washed once with phosphate-buffered saline (PBS). The cell sediment was lysed on ice with cell lysis buffer for $30 \mathrm{~min}$, then $5 \mathrm{X}$ loading buffer was added into the cells, and the mixture was boiled for $10 \mathrm{~min}$ and centrifuged at $12,000 \mathrm{x} \mathrm{g}$ and $20^{\circ} \mathrm{C}$ for $5 \mathrm{~min}$, followed by $10 \%$ sodium dodecyl sulfate polyacrylamide gel electrophoresis (SDS-PAGE) under the voltage of $120 \mathrm{~V} 8 \mu \mathrm{l}$ per lane. After electrophoresis, the target protein was transferred onto a polyvinylidene fluoride (PVDF) membrane, sealed with $5 \%$ skim milk powder for $30 \mathrm{~min}$, and incubated with HIF- $1 \alpha$ and $\beta$-actin antibodies (1:800) for $12 \mathrm{~h}$. The next day, the protein was washed with PBS-Tween-20 3 times ( $5 \mathrm{~min} / \mathrm{time}$ ), and incubated with goat anti-mouse secondary antibody $(1: 1,000)$ at room temperature for $1 \mathrm{~h}$. Finally, a PVDF membrane was coated with luminescent solution (Beyotime, Shanghai, China) for image development. The gray value of the target protein band was scanned by Quantity One software (Bio-Rad Laboratories, Inc., Hercules, CA, USA), and the expression intensity was indicated by the ratio of the target protein band to $\beta$-actin band.

Transwell assay for cell migration. After digestion of the control cell lines and lincRNa-p21 overexpression cell lines, the cell density was adjusted to $5 \times 10^{5}$ cells $/ \mathrm{ml}$ and inoculated into the Transwell upper chamber at $100 \mu \mathrm{l} /$ well. Then, normal fresh medium was added to the lower chamber, incubated in a $37^{\circ} \mathrm{C} 5 \% \mathrm{CO}_{2}$ incubator for $24 \mathrm{~h}$. Then the cells were fixed with paraformaldehyde. After that, the cells in the upper chamber were removed, stained with crystal violet (Sigma-Aldrich; Merck KGaA, Darmstadt, Germany), and washed with tap water after $5 \mathrm{~min}$. The Transwell membrane was dried at room temperature, and then, the invasive ability of the control and observation groups was observed under a microscope (Olympus, Tokyo, Japan).

Detection of cell proliferation via $C C K-8$. The concentrations of control cell lines and lincRNA-p21 overexpression cell lines were adjusted into $5 \times 10^{5}$ cells $/ \mathrm{ml}$, and the cells were inoculated into a 96-well plate (100 $\mu \mathrm{l}$ per well). Then, $10 \mu \mathrm{l}$ 

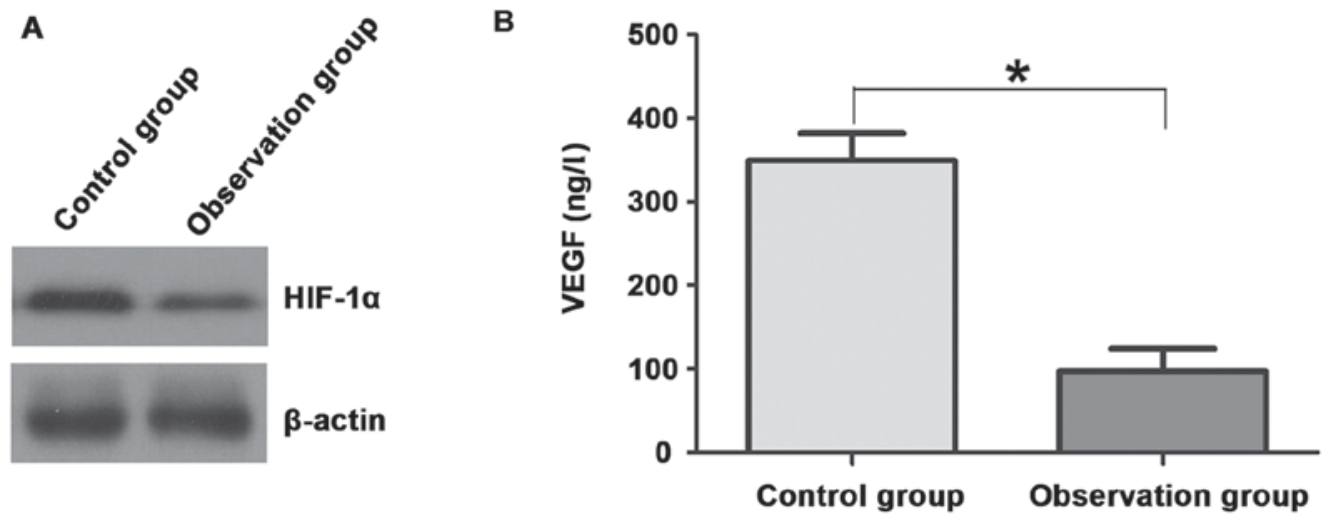

Figure 2. Expression levels of (A) HIF-1 $\alpha$ and (B) VEGF in the control and observation groups. HIF-1 $\alpha$, hypoxia-inducible factor-1 $\alpha$; VEGF, vascular endothelial growth factor. " $\mathrm{P}<0.05$.

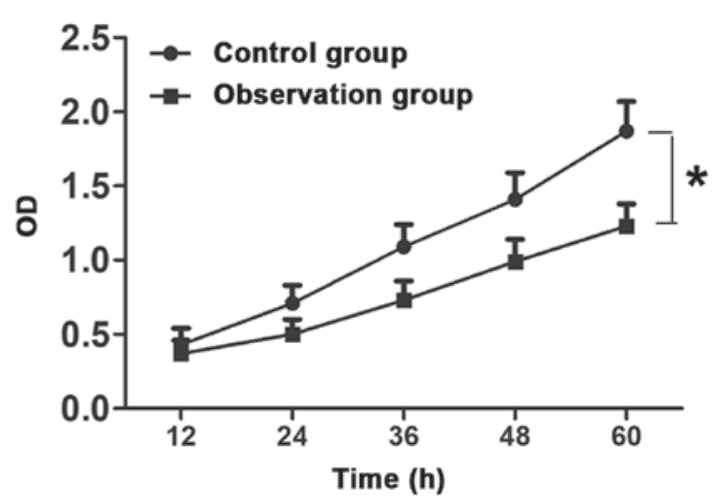

Figure 3. Comparison of cell proliferation capacity between the control and observation groups. ${ }^{*} \mathrm{P}<0.05$.

CCK-8 solution was added into each well at $12,24,36,48$ and $60 \mathrm{~h}$, and the culture plate was incubated for $3 \mathrm{~h}$ at $37^{\circ} \mathrm{C}$, after which the absorbance of each well was measured at a wavelength of $450 \mathrm{~nm}$ by using a microplate reader to analyze cell proliferation.

In vivo tumor formation assay and analysis of number of blood vessels. Control cell lines and lincRNA-p21 overexpression cell lines in the exponential growth phase were digested with trypsin, centrifuged to remove trypsin, and washed once with PBS. Then the cell density was adjusted to $1 \times 10^{8}$ cells $/ \mathrm{ml}$, and inoculated onto the fat pad under left axilla of nude mice (100 $\mu 1$ per mouse). The tumor could be seen at 10 days after inoculation, and at that time the tumor length and width were measured by using a vernier caliper, and the tumor volume was calculated by using the formula. $\mathrm{V}\left(\mathrm{mm}^{3}\right)=1 / 2 \mathrm{x}$ length $\mathrm{x}$ width ${ }^{2}$. The tumor tissues were sectioned, and the number of tumor vessels was analyzed via immunohistochemistry

Tumor vascular analysis. The tumor tissues were fixed with $4 \%$ paraformaldehyde at $4^{\circ} \mathrm{C}$ for $24 \mathrm{~h}$, embedded in paraffin, then sectioned. The slice, $8 \mu \mathrm{m}$ thick, was dewaxed and hydrated, and $0.01 \mathrm{M}$ citrate buffer was added to boil for $10 \mathrm{~min}$ for the repair of antigen. The endogenous catalase was removed by treatment with $0.3 \%$ hydrogen peroxide for $30 \mathrm{~min}$ at room temperature. The tissue was blocked with $10 \%$ goat serum for $30 \mathrm{~min}$, and the rabbit anti-mouse CD31 primary antibody (1:100) was diluted with blocking buffer. Then, it was incubated at $4^{\circ} \mathrm{C}$ for $12 \mathrm{~h}$ and washed 3 times with PBS for 5 min each time. Then, HRP-labeled goat anti-rabbit secondary antibody was diluted $(1: 1,000)$ with blocking buffer, incubated at room temperature for $12 \mathrm{~h}$ and washed 3 times with PBS for 5 min each time. DAB staining was performed,
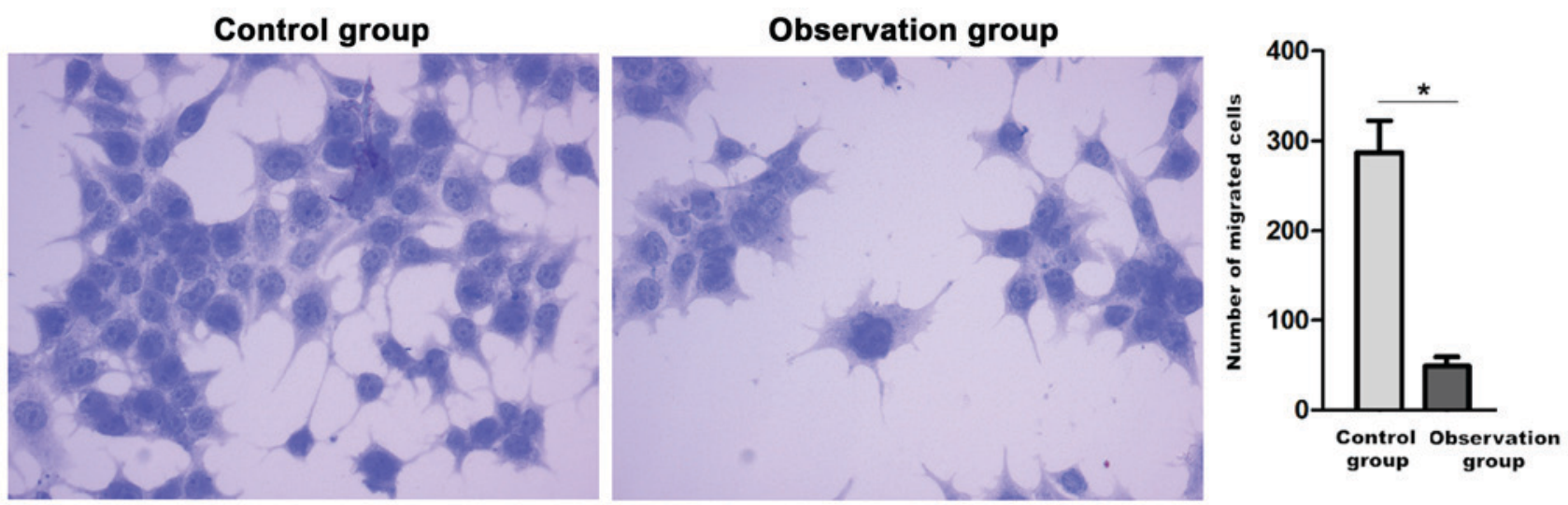

Figure 4. Effect of lincRNA-p21 on invasion capacity of liver cancer cells. The migrated cells in the control and observation groups (magnification, x200). ${ }^{*} \mathrm{P}<0.05$ indicates that the difference was statistically significant. lincRNA, long intergenic non-coding ribonucleic acid. 


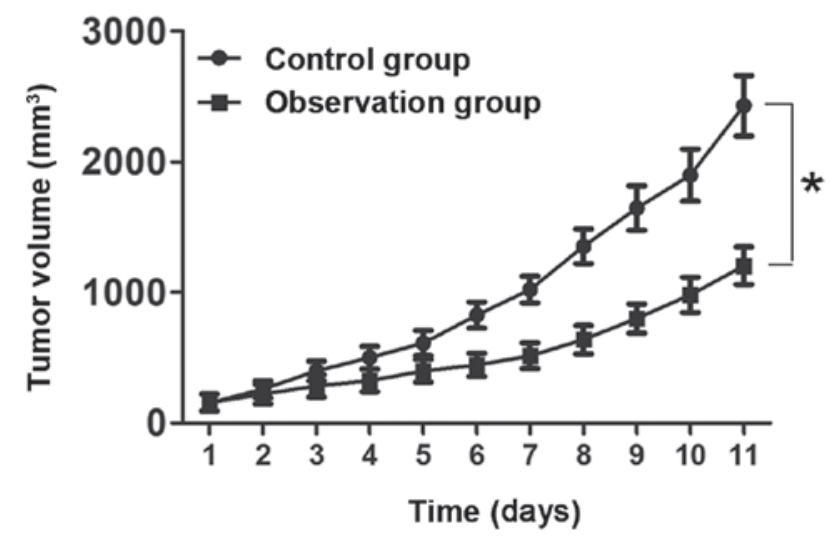

Figure 5. Effect of lincRNA-p21 on in vivo tumor formation of liver cancer cells. ${ }^{*} \mathrm{P}<0.05$. lincRNA, long intergenic non-coding ribonucleic acid.

and the nucleus was stained with hematoxylin. The slice was sealed with neutral resin, and the number of tumor vessels was analyzed via immunohistochemistry.

Statistical analysis. Statistical Product and Service Solutions (SPSS) 13.0 software (SPSS, Inc., Chicago, IL, USA) was used for analysis. Measurement data are presented as mean \pm standard deviation. Student's t-test was used for the comparison of measurement data. $\mathrm{P}<0.05$ indicates that the difference was statistically significant.

\section{Results}

Construction of lincRNA-p21 overexpression liver cancer cell lines. MHCC97H liver cancer cell lines were infected with lentivirus expressing lincRNA-p21, and the empty vector was used as the control. After $48 \mathrm{~h}$ of cell infection, the uninfected cells were killed using puromycin, and the surviving cells were successfully infected. Then the expression level of lincRNA-p21 mRNA was analyzed via RT-qPCR. The results revealed that compared with that in the control group $(1.32 \pm 0.23)$, the expression level of lincRNA-p21 mRNA in the observation group was significantly increased $(8.39 \pm 1.09)$, and there was a statistically significant difference $(\mathrm{P}<0.05)$ (Fig. 1).

Effects of lincRNA-p21 on expression of HIF-1 $\alpha$ and VEGF in liver cancer cells. It was found via bioinformatics that HIF-1 $\alpha$ may be a target gene of lincRNA-p21. Western blotting analysis showed that compared with that in control group, the expression level of HIF-1 $\alpha$ in cells in the observation group was significantly downregulated, and the difference was statistically significant $(\mathrm{P}<0.05)$. Furthermore, the VEGF expression level in cells was analyzed via ELISA, and results showed that compared with that in the control group, the expression level of VEGF in cells in the observation group was also obviously downregulated, and there was a statistically significant difference $(\mathrm{P}<0.05)$ (Fig. 2).

Effect of lincRNA-p21 on proliferation of liver cancer cells. According to the CCK-8 results, compared with that in the control group, the cell proliferation capacity at each time-point in the observation group was obviously reduced, and the difference was statistically significant $(\mathrm{P}<0.05)$ (Fig. 3).
Effect of lincRNA-p21 on invasion of liver cancer cells. The invasion capacity of MHCC97H cells was analyzed via Transwell assay. The cell invasion capacity in the control group (287.32 \pm 35.12$)$ was markedly increased compared with that in the observation group $(49.33 \pm 9.84)$, showing a statistically significant difference $(\mathrm{P}<0.05)$ (Fig. 4).

Effect of lincRNA-p21 on in vivo tumor formation of liver cancer cells. To further verify the effect of lincRNA-p21 overexpression on the tumor formation capacity of liver cancer cells, control cell lines and lincRNA-p21 overexpression cell lines were inoculated subcutaneously in mice. Results showed that the tumor growth rate of control cell lines was obviously higher than that of cells in the observation group, and the difference was statistically significant $(\mathrm{P}<0.05)$. Compared with that in the control group, the number of blood vessels in tumor tissues of mice in the observation group was significantly reduced, and there was a statistically significant difference $(\mathrm{P}<0.05)$ (Fig. 5).

\section{Discussion}

LincRNA-p21 is a tumor suppressor involved in the progression and recurrence of a variety of tumors (11-14). Current studies have found that lincRNA-p21 is significantly downregulated in non-small cell lung cancer tissues, which is not associated with p53 gene mutation (15). It has also been reported that compared with that in normal colon tissues, lincRNA-p21 is significantly downregulated in colon cancer tissues, and p53 gene mutation has no correlation with lincRNA-p21, either (16). Studies have revealed that the expression level of lincRNA-p21 in liver cancer tissues is obviously decreased, and the high expression of lincRNA-p21 can inhibit tumor infiltration through mediating epithelial-mesenchymal transition by Notch signaling pathway. In this study, it was also found that the expression level of lincRNA-p21 in liver cancer tissues was significantly downregulated, and correlation analyses manifested that the expression level of lincRNA-p21 was closely related to tumor vessels. How lincRNA-p21 regulates tumor vessels is a pending question.

The possible targets of lincRNA-p21 were analyzed via bioinformatics, and results indicated that HIF-1 $\alpha$ might be one of the targets of lincRNA-p21. HIF-1 $\alpha$ is one of the HIF, which can initiate the transcription of many target genes under hypoxic conditions, and play an important role in cell tissue development, physiological stress and pathological process $(17,18)$. In solid tumors, HIF-1 $\alpha$ is activated due to acidic and low-oxygen environment in tumor tissues, thus activating the downstream effector molecule VEGF. VEGF can promote neovascularization, providing necessary conditions for tumor growth and metastasis $(19,20)$. In this study, lincRNA-p21 was overexpressed in the highly metastatic liver cancer cell line MHCC97H. Results revealed that the expression levels of HIF-1 $\alpha$ protein and VEGF were obviously downregulated, indicating that HIF-1 $\alpha$ is one of the targets of lincRNA-p21, and the downregulation of HIF-1 $\alpha$ leads to decrease of VEGF level. In addition, it was demonstrated that overexpression of lincRNA-p21 remarkably inhibited the proliferation of MHCC97H cells and reduced the cell invasion capacity. In in vivo tumor formation assay, the growth rate of cells with 
lincRNA-p21 overexpression in vivo significantly declined, which was consistent with the in vitro experimental result.

In conclusion, lincRNA-p21 can reduce the VEGF level through targeting HIF-1 $\alpha$, thereby inhibiting cell proliferation and invasion capacities.

\section{Acknowledgements}

Not applicable.

\section{Funding}

No funding was received.

\section{Availability of data and materials}

The datasets used and/or analyzed during the present study are available from the corresponding author on reasonable request.

\section{Authors' contributions}

YY conceived the study and drafted the manuscript. YP and WL helped with construction of lincRNA-p21 overexpression stable cell lines. YX and CL performed the PCR. CL was responsible for western blotting, and YL contributed to CCK-8 assay. All authors read and approved the final manuscript.

\section{Ethics approval and consent to participate}

The study was approved by the Ethics Committee of Sun Yat-sen Memorial Hospital, Sun Yat-sen University (Guangzhou, China).

\section{Patient consent for publication}

Not applicable.

\section{Competing interests}

The authors declare that they have no competing interests.

\section{References}

1. Zhou Y, Ren H, Dai B, Li J, Shang L, Huang J and Shi X: Hepatocellular carcinoma-derived exosomal miRNA-21 contributes to tumor progression by converting hepatocyte stellate cells to cancer-associated fibroblasts. J Exp Clin Cancer Res 37: 324, 2018.

2. Liao ZB, Tan XL, Dong KS, Zhang HW, Chen XP, Chu L, and Zhang BX: miRNA-448 inhibits cell growth by targeting BCL-2 in hepatocellular carcinoma. Dig Liver Dis: Sep 28, 2018 (Epub ahead of print).

3. Pei Y, Sun X, Guo X, Yin H, Wang L, Tian F, Jing H, Liang X, $\mathrm{Xu} \mathrm{J}$ and Shi P: FGF8 promotes cell proliferation and resistance to EGFR inhibitors via upregulation of EGFR in human hepatocellular carcinoma cells. Oncol Rep 38: 2205-2210, 2017.
4. Huarte M, Guttman M, Feldser D, Garber M, Koziol MJ, Kenzelmann-Broz D, Khalil AM, Zuk O, Amit I, Rabani M, et al: A large intergenic noncoding RNA induced by p53 mediates global gene repression in the p53 response. Cell 142: 409-419, 2010.

5. Talkowski ME, Maussion G, Crapper L, Rosenfeld JA, Blumenthal I, Hanscom C, Chiang C, Lindgren A, Pereira S, Ruderfer D, et al: Disruption of a large intergenic noncoding RNA in subjects with neurodevelopmental disabilities. Am J Hum Genet 91: 1128-1134, 2012.

6. Pan Y, Li C, Chen J, Zhang K, Chu X, Wang R and Chen L: The emerging roles of long noncoding RNA ROR (lincRNA-ROR) and its possible mechanisms in human cancers. Cell Physiol Biochem 40: 219-229, 2016.

7. Zhou P, Sun L, Liu D, Liu C and Sun L: Long non-coding RNA lincRNA-ROR promotes the progression of colon cancer and holds prognostic value by associating with miR-145. Pathol Oncol Res 22: 733-740, 2016.

8. Dimitrova N, Zamudio JR, Jong RM, Soukup D, Resnick R, Sarma K, Ward AJ, Raj A, Lee JT, Sharp PA, et al: LincRNA-p21 activates $\mathrm{p} 21$ in cis to promote Polycomb target gene expression and to enforce the G1/S checkpoint. Mol Cell 54: 777-790, 2014.

9. Yang N, Fu Y,Zhang H, Sima H,Zhu N and Yang G: LincRNA-p21 activates endoplasmic reticulum stress and inhibits hepatocellular carcinoma. Oncotarget 6: 28151-28163,2015.

10. Livak KJ and Schmittgen TD: Analysis of relative gene expression data using real time quantitative PCR and the 2(-Delta Delta C(T)) method. Methods 25: 402 408, 2001.

11. Wang G, Li Z, Zhao Q, Zhu Y, Zhao C, Li X, Ma Z, Li X and Zhang Y: LincRNA-p21 enhances the sensitivity of radiotherapy for human colorectal cancer by targeting the Wnt/ $\beta$-catenin signaling pathway. Oncol Rep 31: 1839-1845, 2014.

12. Jiang YJ and Bikle DD: LncRNA profiling reveals new mechanism for VDR protection against skin cancer formation. J Steroid Biochem Mol Biol 144: 87-90, 2014

13. Işın M, Uysaler E, Özgür E, Köseoğlu H, Şanlı Ö, Yücel ÖB, Gezer U and Dalay N: Exosomal lncRNA-p21 levels may help to distinguish prostate cancer from benign disease. Front Genet 6: 168, 2015.

14. Zhai H, Fesler A, Schee K, Fodstad O, Flatmark K and Ju J: Clinical significance of long intergenic noncoding RNA-p21 in colorectal cancer. Clin Colorectal Cancer 12: 261-266, 2013.

15. Castellano JJ, Navarro A, Viñolas N, Marrades RM, Moises J, Cordeiro A, Saco A, Muñoz C, Fuster D, Molins L, et al: LincRNA-p21 impacts prognosis in resected non-small cell lung cancer patients through angiogenesis regulation. J Thorac Oncol 11: 2173-2182, 2016.

16. JiaM,JiangL,Wang YD,HuangJZ,YuMandXueHZ:lincRNA-p21 inhibits invasion and metastasis of hepatocellular carcinoma through Notch signaling-induced epithelial-mesenchymal transition. Hepatol Res 46: 1137-1144, 2016.

17. Schofield CJ and Ratcliffe PJ: Oxygen sensing by HIF hydroxylases. Nat Rev Mol Cell Biol 5: 343-354, 2004.

18. Bartrons R and Caro J: Hypoxia, glucose metabolism and the Warburg's effect. J Bioenerg Biomembr 39: 223-229, 2007.

19. Yu J, Liang F, Huang H, Pirttiniemi P and Yu D: Effects of loading on chondrocyte hypoxia, HIF-1 $\alpha$ and VEGF in the mandibular condylar cartilage of young rats. Orthod Craniofac Res 21: 41-47, 2018.

20. Dewangan J, Kaushik S, Rath SK and Balapure AK: Centchroman regulates breast cancer angiogenesis via inhibition of HIF-1 $\alpha /$ VEGFR2 signalling axis. Life Sci 193: 9-19, 2018.

This work is licensed under a Creative Commons Attribution-NonCommercial-NoDerivatives 4.0 International (CC BY-NC-ND 4.0) License. 